\title{
DISKS IN THE PRE-MAIN SEQUENCE BINARY ENVIRONMENT
}

\author{
ROBERT D. MATHIEU \\ Department of Astronomy \\ University of Wisconsin \\ Madison, Wisconsin 53706 USA
}

\begin{abstract}
The interaction of disks and young binary stars is discussed, with particular emphasis on disk structure, the existence of accretion at the stellar surfaces and binary orbital evolution.
\end{abstract}

\section{Pre-Main Sequence Binaries and Disks - Overview}

Low-mass pre-main sequence (PMS) binaries have been known since the earliest studies of T Tauri stars. However, prior to a decade ago, all of the known PMS binaries were visual pairs. Given that the nearest star-forming regions are roughly $150 \mathrm{pc}$ distant, these binaries have separations greater than $100 \mathrm{AU}$. This restriction to wide binaries is significant in that $100 \mathrm{AU}$ is also the typical value cited for the outer radii of accretion disks around young stars. Fortunately, in the past decade the first PMS spectroscopic binaries have been discovered and technological advances (lunar occultation, speckle interferometry, slit scan, infrared imaging) have permitted angular resolution of binaries with separations as small as $1 \mathrm{AU}$. These binaries have provided a window on binary-disk interactions in the young binary environment.

It is not the purpose here to give a complete review of PMS binaries, for which the reader is referred to Bodenheimer, Ruzmaikina and Mathieu (1991), Zinnecker (1990) and Reipurth (1988). Nonetheless, two particularly important points are worth noting. Presently, there is no indication that the PMS binary frequency as a function of period differs from that found among solar-mass main sequence (MS) stars for periods of a few days to $10^{4}$ yr (Mathieu, Walter and Myers 1989, Simon et al. 1991). Thus, by the PMS stage of evolution the MS period distribution is largely established. This assessment must be taken with care, however, as the PMS binary surveys have not been as extensive as those for MS stars. Differences in frequency of a factor two over several decades in period would be only marginally detectable with the data in hand. Thus, for example, there remains room for orbital period evolution during the PMS evolution of a binary.

Secondly, PMS binaries of all periods have been found throughout the PMS domain of the H-R diagram, including near the stellar birthline. Thus some binaries (and perhaps all, given the similar MS and PMS binary frequencies) are formed prior to the PMS stage of evolution. If the evolutionary timescales of stars in binaries are like those derived theoretically for single stars, the binary formation timescale is less than $\approx 10^{5} \mathrm{yr}$.

To the best of our knowledge, all of the presently known PMS binaries are detached systems. Nonetheless, solar-mass stars at the stellar birthline would fill the Roche lobes of the shortest-period binaries. Furthermore, in the broad definition of "interacting" used at this meeting, some of the known PMS binaries must indeed be 
interacting. For example, the classical T Tauri stars are thought to have strong winds which must interact with companions. And certainly in the shortest period systems significant tidal interactions are occurring. The lack of evidence for PMS interacting binaries is likely an indication that we have not yet looked with sufficient care, and possibly that the signatures of interaction are confused with those of T Tauri activity.

Perhaps the most common and important interaction in PMS binaries, however, is not between the two stars but rather between the binary and an associated disk. Indeed, in young binaries this interaction is rather unique, since at least initially the disk has a source of material external to the stars (e.g., a dense molecular core). Thus:

- For many young binary systems, the outer radius of an associated disk may be substantially larger than the binary semimajor axis. In studies of single PMS stars, a typical estimate for the outer radius of a disk is 100 AU. For solar-mass stars a binary with comparable semimajor axis would have a period of $700 \mathrm{yr}$, longer than the median period of $180 \mathrm{yr}$ for MS binaries (Duquennoy and Mayor 1991). Thus, if such disk radii are also typical for binary systems, at some point in their early evolution most binaries are embedded within their associated disks.

- The existence and evolution of the disk is not primarily linked to the evolutionary states of the stars. For example, since the source of the disk material is independent of the stars, the disk mass is not tied to stellar evolution (in contrast to giants overflowing their Roche lobes, for example). Important indirect connections exist, however. For example, energetic outflows which may be linked to stellar evolution impact on the surrounding reservoir of material feeding the disk, and the radiation fields of the stars certainly influence the composition and temperature structure of the disk and thus its evolution.

This paper takes an observational perspective on three issues related to the interaction between young binaries and their associated disks: disk structure in the young binary environment, the existence of accretion at the stellar surfaces and binary orbital evolution. While at this early stage the goal is primarily to develop a framework of facts within which ideas can develop, ultimately these issues are fundamental to understanding the primary branch of the star-formation process, the formation of binary stars.

\section{Observations of Disk Structure in Young Binaries}

The structure of disks in the young binary environment is certainly more complicated than that of disks around young single stars. For large primary-secondary mass ratio, the disk is nearly axisymmetric about the primary. Dynamical theory argues that the companion will drive an annular gap in the disk at its orbital radius (e.g., Lin and Papaloizou 1991). For mass ratios nearer unity, the distribution of material is only now being studied theoretically; as a starting point for discussion, the disk may be considered as three discrete coplanar components: circumprimary and circumsecondary (disks associated with each star and having size scales smaller than the orbital semimajor axis; together referred to as circumstellar disks) and circumbinary (a disk surrounding the binary, external to the binary orbit). The observational challenge is to determine the evolutionary histories of these components.

One of the most intriguing recent observational results has been the millimeter studies of Beckwith, Sargent and their collaborators (Beckwith and Sargent 1991, Beckwith et al. 1990). For typical disk models, much of the disk is optically thin at millimeter wavelengths. Thus, with adopted temperature distributions derived from the infrared spectral energy distributions, estimates of disk masses can be derived. These 
investigators have observed at $1.3 \mathrm{~mm}$ a sample of 17 PMS binaries, most having projected separations between $1 \mathrm{AU}$ and $1000 \mathrm{AU}$. They found detectable disk masses $\left(\mathrm{M}_{\text {disk }} \gtrsim 0.01 \mathrm{M}_{\mathrm{o}}\right)$ only among those binaries with projected separations greater than $\approx 100$ AU, comparable to the nominal outer radii of disks around PMS stars. Thus one intriguing implication might be that binaries embedded within disks act to reduce the total disk mass through a dynamical mechanism. It is also possible that the formation process itself reduces the disk mass, for example through exhaustion in forming the companion from the disk or in expulsion of disk material during capture of the companion.

As Beckwith and Sargent (1991) point out, their upper limits on the masses of disks associated with binaries having separations less than $100 \mathrm{AU}$ are similar or only slightly smaller than the detected masses. Also, Leinert et al. (1991) have recently found GG Tau to be a binary with projected separation of $40 \mathrm{AU}$; Beckwith et al. (1990) found the disk associated with GG Tau to have a disk mass of $0.3 \mathrm{M}_{\mathrm{O}}$, one of their largest derived disk masses. Similarly, Weintraub (private communication) has found GW Ori (separation $\approx 1 \mathrm{AU}$ ) to have a strong millimeter flux. Thus the suggested dependence of disk mass on binary separation needs to be put on firmer footing.

The detailed structure of disks associated with young binaries can be probed by analysis of the infrared spectral energy distributions (SED's) of PMS binaries. For example, a binary with a large primary-secondary mass ratio may have optically thick circumprimary and circumbinary disks, separated by an annular optically thin gap. In this case, a decrease in flux would be expected at that wavelength characteristic of the temperature at the radius of the gap. As another example, a system having only a circumbinary disk would have a large far-infrared excess relative to that in the near infrared. This approach is particularly powerful for studying the structure of disks in short-period binaries (semimajor axes of roughly one AU or less), since such stellar separations correspond to the size scales in disks from which the continuum emission is primarily at wavelengths less than $20 \mu \mathrm{m}$ (i.e., observationally accessible from the ground). Also, $10 \mu \mathrm{m}$ silicate emission might be expected from optically thin regions on such size scales, thus providing an independent diagnostic of complex disk structure.

This approach has been applied to the spectral energy distribution of GW Ori, a classical T Tauri star with strong $\mathrm{H} \alpha$ emission and excess infrared luminosity comparable to the photospheric luminosity. GW Ori is a single-lined spectroscopic binary with a period of 242 days, corresponding to a separation of the components of slightly greater than $1 \mathrm{AU}$ and a mass ratio $\approx 3$ (Mathieu, Adams and Latham 1991; MAL). The spectral energy distribution of GW Ori is shown in Figure 1. The dip in the energy distribution

Figure 1. The spectral energy distribution of the $\mathrm{T}$ Tauri binary GW Ori. The open boxes are derived from measured fluxes. The solid curve is a theoretical model derived from a spatially thin, optically thick disk from which an annular gap has been removed. The dashed curve shows the same model without the gap.

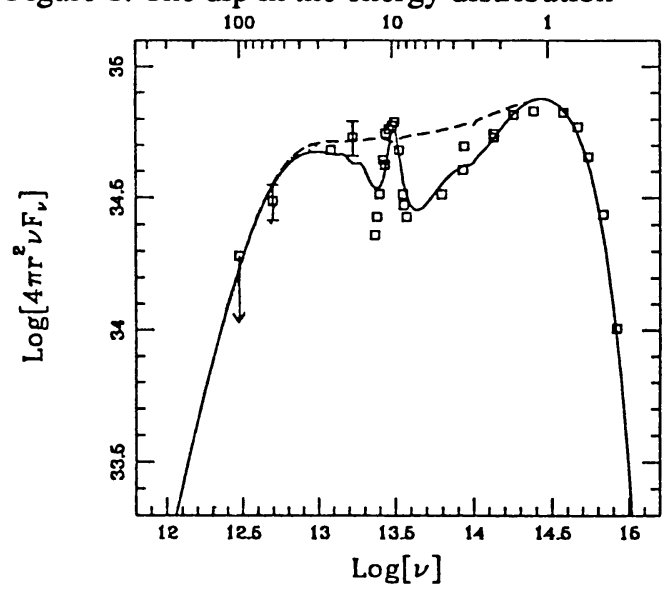


between $2 \mu \mathrm{m}$ and $20 \mu \mathrm{m}$ and the strong silicate emission feature at $10 \mu \mathrm{m}$ are notable.

MAL interpreted the structure in the flux distribution as due to a nearly evacuated annular gap in an otherwise optically thick disk. The near-infrared excess is provided by at least one circumstellar disk (taken to be circumprimary) while the far-infrared emission is attributed to a circumbinary disk. The silicate emission is attributed to optically thin hot dust in the gap between the two disks. The spectral energy distribution of such a configuration has been computed by MAL for an axisymmetric, spatially thin disk with a power-law radial temperature distribution, shown by the curve in Figure 1 . They find that a disk with a gap from $0.17 \mathrm{AU}$ to $3.3 \mathrm{AU}$ provides a reasonable match to the observed flux distribution, and is consistent with the independent constraint that the the companion orbit lie within the gap. As with all "flat spectrum" young stellar objects, the large disk luminosity, particularly in the far infrared, is difficult to produce through accretion mechanisms, as discussed in detail by MAL. This problem can be relieved somewhat by attributing the far-infrared emission to reprocessed photospheric light from a shell at distances greater than $200 \mathrm{AU}$ from the binary. Even so, the energy budget is such that the entire excess infrared luminosity cannot be explained as reprocessed photospheric light, suggesting active accretion in the disk.

Other short-period PMS binaries also show evidence for associated disks in their infrared SED's; four are shown in Figure 2. All have both near- and far-infrared excesses, indicating both circumstellar and circumbinary material. Two of the four show marked changes in their spectral slopes between $3.5 \mu \mathrm{m}$ and $10 \mu \mathrm{m}$. One case of particular interest is the binary 162814-2427. In this case, the slope of the spectral energy distribution around $2 \mu \mathrm{m}$ is too steep for an optically thick disk, even if only a passive reprocessing disk, and thus indicates an optically thin inner region of the disk. However, the $10 \mu \mathrm{m}$ and $20 \mu \mathrm{m}$ excesses indicate that the disk is optically thick at larger radii. Together these data argue for an optically thick disk with an inner hole. The theoretical curve shown with the observations of 162814-2427 is derived from a preliminary model having an axisymmetric, spatially thin, optically thick disk with an optically thin region interior to a radius of $0.3 \mathrm{AU}$ (Jensen et al. 1991). The independently derived semimajor axis of the orbit of 162814-2427 is also $0.3 \mathrm{AU}$ (to within a factor of $\sin ^{3} \mathrm{i}$, which is likely near
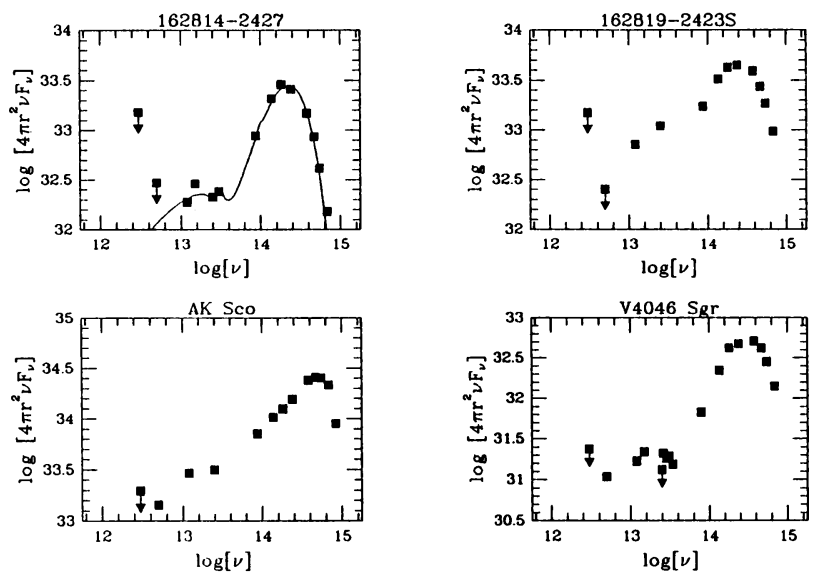

Figure 2. The spectral energy distributions of four pre-main sequence spectroscopic binaries (data from literature). The solid curve fit to the observations of 162814-2427 is a theoretical model derived from a spatially thin, optically thick disk with a central hole. 
unity (Mathieu, Walter and Myers 1989)). Thus, while this model is clearly only a crude approximation to the actual distribution of the disk material, it does suggest that the existence and size of the inner hole in the disk may be set by the binary itself. More generally, the system may indicate that circumstellar disks disappear prior to circumbinary disks.

One possible mechanism for the creation of such an inner hole is the binary acting dynamically to restrict accretion from the circumbinary disk to the circumstellar disks (e.g., Artymowicz et al. 1991). Thus the circumstellar disks, continuing to accrete but insufficiently replenished, would become largely exhausted. As shown in the next section, there is evidence that accretion continues near at least one stellar surface in 162814-2427, but at a slow rate. This, and the presence of optically thin emission, may indicate that some material continues to leak inward from the circumbinary disk to the circumstellar region. The orbit of $162814-2427$ is quite eccentric $(e=0.48)$, which may promote such leakage. (For a related discussion of central holes in disks around apparently single stars, see Skrutskie et al. 1990 who suggest that in these cases the holes may result from the action of planet formation.)

Finally, the discovery of binaries with infrared companions (e.g., Zinnecker 1990) should caution against consideration of only simple coplanar disk-like distributions of extended material in young binary environments. These binaries, in which the more luminous component is only detectable in the infrared while the companion is optically visible, may be indicative of more complex geometries during the evolution of young binaries.

\section{Accretion in the Young Binary Environment}

At present, our picture of the star formation phenomenon can be characterized as an accretion process. Indirect evidence suggests active accretion onto young, (apparently) single stars. Our understanding of the role of accretion in the binary formation process is nil, but certainly it is of interest to assess whether accretion onto stellar surfaces continues in young binaries. More specifically, the discussion here will focus on the presence in young binaries of observable diagnostics of active accretion.

Historically, strong $\mathrm{H} \alpha$ emission was a primary detection criterion for young stars. As noted above, many of the classical T Tauri stars with strong $\mathrm{H} \alpha$ emission have long been known to be visual binaries, and more recently others have been found to be binaries with separations as small as 1 AU. Indeed, Simon et al. (1991) have argued that $\mathrm{H} \alpha$ emission strengths are similar for single stars and for binaries with separations greater than a few AU. Recently it has been argued that $\mathrm{H} \alpha$ emission strength correlates with accretion rate, as determined from other diagnostics (see discussion of Skrutskie et al. 1990). If so, then significant accretion rates can occur in the young binary environment.

Interestingly, however, only one short-period ( $\mathrm{P}<<1 \mathrm{yr}) \mathrm{PMS}$ binary has been found to have strong $\mathrm{H} \alpha$ emission (V4046 Sgr; de la Reza et al. 1986), while more than ten short-period binaries have been found among PMS stars with weak or no H $\alpha$ emission (see also the discussion in Mathieu, Walter and Myers 1989). This may indicate that close binaries reduce accretion rates or disrupt the $\mathrm{H} \alpha$ emission process directly. As $\mathrm{H} \alpha$ emission is thought to arise on size scales of a few stellar radii, much smaller than the separations of most short-period binaries, it seems more likely that the influence of such binaries on $\mathrm{H} \alpha$ emission would be indirect through their influence on the disk. Perhaps, as suggested by the binary 162814-2427 discussed in the last section, the binary acts to diminish the circumstellar disks, reduce the accretion rate and thus starve the $\mathrm{H} \alpha$ emission mechanism. The question remains, however, as to why only short-period systems would have this effect; the answer may be related to the interplay of available material in 
circumstellar disks and accretion timescales.

A second diagnostic for active accretion at a stellar surface is the presence of ultraviolet excesses and spectral veiling. Recently, these effects have been attributed to emission from a boundary layer at a stellar surface heated by accretion (e.g., Bertout, Basri and Bouvier 1988). One well studied case is DF Tau, a heavily veiled classical T Tauri star. Based on the excess ultraviolet light, Bertout, Basri and Bouvier (1988) derived an accretion rate of a few times $10^{-7} \mathrm{M}_{\mathrm{o}} \mathrm{yr}^{-1}$, typical among actively accreting $\mathrm{T}$ Tauri stars. They also found the infrared spectral energy distribution to be consistent with a viscous accretion disk. Lastly, they found periodic light variation with increasing amplitude toward the blue, which they interpreted as a hot spot on the surface of the star due to accretion along magnetic field lines. These results are relevant here in that DF Tau is a spatially resolved binary with projected separation of $60 \mathrm{AU}$ (Simon et al. 1991). Again, this evidence argues that substantial accretion can occur at a stellar surface in a young binary. MAL also argue for large accretion rates in the GW Ori system; the excess near-infrared luminosity alone requires accretion rates of $10^{-6} \mathrm{M}_{\mathrm{o}} \mathrm{yr}^{-1}$. Interestingly, though, GW Ori shows no evident spectral veiling or ultraviolet excess.

A third diagnostic for accretion is the $\mathrm{H} \alpha$ emission line profile. Recently, Jensen $e t$ al. (1991) have found the binary 162814-2427 to show an inverse-P-Cygni H $\alpha$ profile. Typically such profiles are taken as evidence of infalling material near the stellar surface

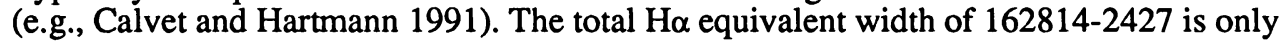
a few Angstroms in emission, so the accretion rate is likely low -- consistent with the lack of circumstellar disks, as argued in the previous section. Numerous spectra acquired to date show that the $\mathrm{H} \alpha$ line profile may vary with orbital phase. If confirmed by further observation, this binary raises the possibility of recovering the detailed spatial distribution and kinematics of the accretion flow.

To summarize, observable diagnostics attributed to active accretion -- strong $\mathrm{H} \alpha$ emission, ultraviolet excesses, spectral veiling, inverse-P-Cygni line profiles -- have been found in PMS binaries, particularly among the longer period systems. Apparently accretion onto stellar surfaces can occur in the young binary environment. If the accreting material at the stellar surface is presumed to derive solely from a circumstellar disk, then the critical question is whether such disks can be replenished, perhaps with material from a circumbinary disk. If not (as found theoretically by Artymowicz et al. (1991) for a circumbinary disk around a near-circular binary), then one might anticipate that shorter period binaries would be less likely to show large accretion rates since their less extensive circumstellar disks would be more quickly exhausted.

\section{The Eccentricity Distribution of Pre-Main Sequence Binaries}

The discussion so far has considered evidence for the dynamical influence of a young binary on an associated accretion disk. Of course, a dynamical interaction must be reciprocal and result in evolution of the binary orbital elements as well. Again, GW Ori (Section 2) plays a central role in motivating this discussion and providing insight into the influence of disks on binary orbits. MAL find the orbit of GW Ori to be very nearly circular at an orbital period of 242 days. ${ }^{1}$ This result is to be compared with the eccentricity distribution as a function of orbital period for main sequence solar-mass stars (Figure 3, derived from the results of Duquennoy and Mayor 1991). Circular orbits among main sequence binaries with periods of greater than about ten days are rare

$\overline{1}$ Formally the measured eccentricity $(e=0.04 \pm 0.06)$ is indistinguishable from zero, but the measurement error is large enough that it would be premature to assert definitively that the orbit is circular. 


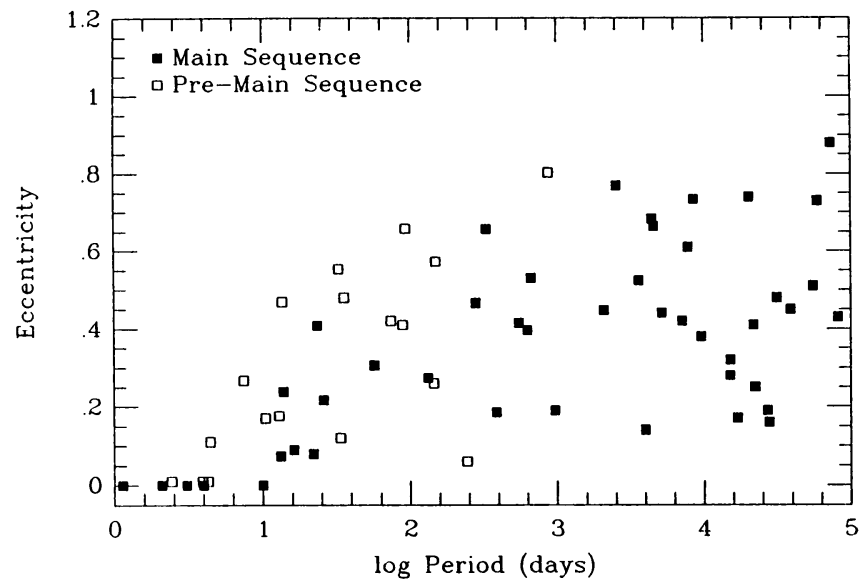

Figure 3. The period-eccentricity distribution for main sequence solar-mass binaries (filled; Duquennoy and Mayor 1991) and for pre-main sequence binaries (open).

(frequency of a few percent; Griffin 1991). While it is possible that GW Ori is a precursor of such a binary, it is arguably more likely that ultimately GW Ori will also have an eccentric orbit. If so, the case of GW Ori poses two questions: why is the orbit circular at present and what process will drive eccentricity into the orbit?

It is tempting to suggest that the answers to both questions lie with the disk associated with GW Ori. Goldreich and Tremaine (1980; see also Ward 1988) found in the case of planetesimal companions embedded within a disk that orbital evolution would be very rapid, but the sign of the eccentricity evolution was sensitive to the details of the disk structure. Artymowicz et al. (1991) have done numerical experiments with binaries having nearly equal mass stars surrounded only by circumbinary disks. They found that such disks will rapidly drive large eccentricities into near-circular binary orbits. A similar experiment with a circumstellar disk, as also exists in the GW Ori system, remains to be done, although Artymowicz et al. (1991) argue that in this case the eccentricity should again increase. Evidently, however, this increase in eccentricity has not yet occurred in the GW Ori system.

In contrast to GW Ori, the binary $162814-2427$ has an orbital eccentricity of 0.48 . It was argued above that at present this binary has only a circumbinary disk, indicating that the system is in a later stage of disk evolution than GW Ori. Clearly, orbital eccentricity has been established in 162814-2427 prior to complete dispersal of the disk. Considered together, these two anecdotal cases suggest that the eccentricity distribution of main sequence binaries $(P>10$ d) ultimately may be set by the details of disk evolution in the young binary environment.

More generally, the distribution of PMS orbital eccentricity with period is shown in Figure 3 (Mathieu, Marschall and Latham 1991), along with the eccentricity distribution for main sequence (MS) solar-mass binaries. Both the PMS and MS distributions are characterized by circular orbits at the shortest periods and a distribution of orbital eccentricity for longer periods. Notably, the transition period between circular and eccentric orbits is 4 days for PMS binaries and 11 days for MS binaries. Between these circularization cutoff periods and periods of about 1000 days there is an increase of the maximum eccentricity with increasing period. The largest eccentricities are only found at the longest periods. For periods longer than the circularization cutoffs where both PMS and MS data are available, the eccentricity distributions of the PMS and MS binaries are 
not distinguishable. Apparently, as with the orbital period distribution, by the PMS stage of evolution the MS eccentricity distribution is largely established.

The circular orbits of PMS binaries with periods of less than 4 days likely represent an interaction not discussed at length at this symposium, that being stellar tidal interactions leading to circularization of orbits. If the short-period circular orbits are attributed to tidal circularization during the PMS phase, then the transition period between circular and eccentric orbits represents a critical benchmark for the evolution of internal stellar structure and orbital separation prior to $\approx 10^{6} \mathrm{yr}$. In this vein, Zahn and Bouchet (1989) have done a theoretical analysis of the expected tidal circularization as binary components evolve from the stellar birthline to the main sequence. They come to the conclusion that the circularization of a binary orbit takes place almost entirely at the beginning of the Hayashi phase, and that there is little further decrease of orbital eccentricity while on the main sequence. For a variety of assumptions regarding initial conditions and binary component masses, they predict that circularization cutoff periods between 7.2 and 8.5 days should be found for low-mass binaries of all ages. Unfortunately, at present the number of PMS binaries with periods in the critical regime of 4-10 days are limited (see Bodenheimer, Ruzmaikina and Mathieu 1991): EK Cep has an orbital eccentricity of 0.1 at a period of 4.4 days and a second unpublished orbit has an eccentricity of 0.3 at a period of 7.5 days. Given the transition period of 11 days among field solar-mass stars, it would seem difficult to reconcile these two PMS binaries with orbital circularization occurring only near the stellar birthline. However, the observational data are limited. It should also be noted that the stellar tidal circularization theory remains uncertain (e.g., Goldman and Mazeh 1991), with significant impact on estimates of circularization timescales.

At the longest periods $\left(P>1000^{d}\right)$ Duquennoy and Mayor (1991) argue that the MS eccentricity distribution tends toward the dynamically relaxed distribution $f(e)=2 e$. They suggest that stellar dynamical processes (such as encounters in small groups of stars) may have determined the eccentricity distribution of these binaries. ${ }^{2}$ Thus they argue that the only period regime in which binaries have not undergone substantial orbital evolution after binary formation lies between the circularization cutoff and $\approx 1000 \mathrm{~d}$. Averaged over period, the eccentricity distribution in this intermediate period range is "bell-shaped" with a mean eccentricity of 0.35 , which they suggest may be the product of the binary formation process.

However, in detail the eccentricity distribution as a function of period suggests that the converse hypothesis also merits consideration: the long-period MS eccentricity distribution may be the result of the formation process, with evolutionary processes having later removed the most eccentric binaries from the shortest period systems. For periods of less than $1000^{\mathrm{d}}$, both the MS and PMS eccentricity distributions show a decrease in the maximum orbital eccentricity with decreasing period. Indeed, in both samples even intermediate eccentricities are not found for periods immediately above the circularization cutoffs. Arguably this distribution might result from an evolutionary process which acts to reduce orbital eccentricity and which is most effective at short periods and high eccentricity. One such process is stellar tidal circularization, which as Zahn and Bouchet (1989) argue is particularly effective for PMS stars with large radii and deep convective zones. Their work should be extended to consideration of eccentricity distributions at longer periods. Other evolutionary processes should also be at considered, such as mass transfer through wind accretion and the influences of disks.

In truth, it is not clear that the binary formation process can be clearly distinguished from the early evolution of orbital eccentricity or that the orbital evolution can be described

\footnotetext{
$\overline{2}$ It is not clear why such a dynamical process should only act on binaries with periods greater than $\approx 1000$ days, or equivalently semimajor axes of order $1 \mathrm{AU}$ and orbital velocities of order $10 \mathrm{kms}^{-1}$.
} 
in such discrete stages. Many processes are operating simultaneously in the earliest stages of a binary's existence. For example, if binary formation is typically a capture process, it is likely that energy dissipation in disks plays an essential role, so that "formation" and "orbital evolution" occur simultaneously. Similarly, dissipative processes will be active shortly after binary formation through either fragmentation or global disk instabilities. Furthermore, massive disks are present from formation into the PMS stage of evolution; ultimately disk evolutionary timescales may dictate the period-eccentricity distribution. Tidal circularization must indeed occur in the shorter period systems. And stellar encounters may be relevant, at least in cluster environments if not in all dense molecular cores. The problem at hand is to determine which of these processes has the last word in establishing the main sequence eccentricity distribution.

\section{Summary}

The interactions of stars and disks in young binary systems likely play an essential role in establishing the properties of the main sequence binary population. Observational studies of disks associated with young binaries have begun to reveal their structure and suggest possible evolutionary histories for both the disks and the binaries. In particular:

1) Binary star formation occurs prior to the stellar birthline. Furthermore, by the pre-main sequence stage of evolution the orbital period and eccentricity distributions of main sequence solar-mass binaries are already largely established.

2) Infrared excesses and millimeter wavelength emission reveal the presence of extended material associated with some PMS binaries. The existence of both near- and far-infrared excesses indicates the presence of material both within and outside of binary orbits.

3) Infrared spectral energy distributions indicate the extended material associated with young binaries has complex structure on size scales comparable to the stellar separations. If this material is taken to be distributed primarily in disks, than analyses of the spectral energy distributions of several short-period binaries suggest annular gaps or central holes in some disks. Disks in which binaries are embedded may also tend to be less massive than disks associated with single stars or wide binaries.

4) Several lines of evidence indicate that active accretion at stellar surfaces occurs in young binaries. Observable diagnostics of accretion are not found to differ substantially between single stars and binaries with separations greater than a few AU. There is weak evidence that closer binaries may act to inhibit large accretion rates. This evidence of active accretion in young binaries challenges theorists to understand the nature of the accretion process in the binary environment, particularly on size scales comparable to the stellar separations and for eccentric orbits.

5) The early evolution of a binary orbit is closely linked to the mass distribution in the binary environment. The near-circular orbit of GW Ori despite its relatively long period may be evidence that disks play a primary role in determining orbital eccentricity. Indeed, the eccentricity distribution of main sequence binaries ultimately may be set by the details of disk evolution in the young binary environment. Nonetheless, other processes, such as tidal circularization and stellar encounters, must come into play in certain circumstances.

Finally, young binary stars are observable analogs to young planetary systems. Thus the study of disk-star interactions in young binaries has potential implications for the 
formation of planetary systems. For example, such binaries provide testing grounds for dynamical theory similar to that applied to the early evolution of planetary systems. When combined with the direct insights into the formation and early evolution of binaries themselves, the study of disks in young binary environments promises to be an intriguing and rich field of research for the coming decade.

I would like to thank Bill Caplan and Eric Jensen for assistance and critical readings of the manuscript, and gratefully acknowledge the support of National Science Foundation Grant AST8814986, the Presidential Young Investigator program and the International Astronomical Union.

\section{References}

Artymowicz, P., Clarke, C.J., Lubow, S.H., and Pringle, J.E. 1991, Ap.J.L, 370, L35

Beckwith, S.V.W., Sargent, A.I., Chini, R.S., and Gusten, R. 1990, AJ, 99, 924

Beckwith, S.V.W. and Sargent, A.I. 1990, in Protostars and Planets III, edited by M. S. Mathews and E. Levy (University of Arizona Press, Tucson) (in press)

Bertout, C., Basri, G., and Bouvier, J. 1988, ApJ, 330, 350

Bodenheimer, P., Ruzmaikina, T., and Mathieu, R.D. 1991, in Protostars and Planets III, edited by M. S. Mathews and E. Levy (University of Arizona Press, Tucson) (in press)

Calvet, N. and Hartmann, L.W. 1991, ApJ (in press)

de la Reza, R., Quast, G., Torres, C.A.D., Mayor, M., Meylan, G., and Llorente de Andres, F. 1986, in New Insights in Astrophysics (ESA SP263), p. 107

Duquennoy, A. and Mayor, M. 1991, A\&A (in press)

Goldman, I. and Mazeh, T. 1991, ApJ, 276, 260

Goldreich, P. and Tremaine, S. 1980, ApJ, 241, 425

Griffin, R.F. 1991, The Observatory (in press)

Jensen, E.L., Caplan, W., Mathieu, R.D., Lee, C.-W., and Walter, F.M. 1991, in preparation

Leinert, C., Haas, M., Richichi, A., Zinnecker, H., and Mundt, R. 1991, A\&A (in press)

Lin, D.N.C. and Papaloizou, J.P.C. 1991, in Protostars and Planets III, edited by M. S. Mathews and E. Levy (University of Arizona Press, Tucson) (in press)

Mathieu, R.D., Adams, F.C., and Latham, D.W. 1991, AJ, 101, 2184

Mathieu, R.D., Marschall, L.M., and Latham, D.W. 1991, in preparation

Mathieu, R.D., Walter, F.M., and Myers, P.C. 1989, AJ, 98, 987

Reipurth, B. 1988, in Formation and Evolution of Low Mass Stars, edited by A.K. Dupree and M.T.V.T. Lago, (Kluwer, Dordrecht), p. 305

Simon, M., Chen, W.P., Howell, R.R., Benson, J.A., and Slowik, D. 1991, ApJ (in press)

Skrutskie, M.F., Dutkevitch, D., Strom, S.E., Edwards, S.E., and Strom, K.M. 1990, AJ, 99, 1187.

Ward, W.R. 1988, Icarus, 73, 330

Zahn, J.P. and Bouchet, L. 1989, A\&A, 223, 112

Zinnecker, H. 1990, in Low-Mass Star Formation and Pre-Main Sequence Objects, edited by B. Reipurth, (ESO, Munich), p. 447 